\title{
Optical enhancement of room temperature ferromagnetism in Er-doped GaN epilayers
}

\author{
N. Nepal, ${ }^{1, a)}$ J. M. Zavada, ${ }^{1}$ R. Dahal, ${ }^{2}$ C. Ugolini, ${ }^{2}$ A. Sedhain, ${ }^{2}$ J. Y. Lin, ${ }^{3}$ and \\ H. X. Jiang ${ }^{3}$ \\ ${ }^{1}$ Department of Electrical and Computer Engineering, North Carolina State University, Raleigh, \\ North Carolina 27695, USA \\ ${ }^{2}$ Department of Physics, Kansas State University, Manhattan, Kansas 66506, USA \\ ${ }^{3}$ Department of Electrical and Computer Engineering and Nano Tech Center, Texas Tech University, \\ Lubbock, Texas 79409, USA
}

(Received 1 May 2009; accepted 23 June 2009; published online 14 July 2009)

\begin{abstract}
We report on the enhancement of magnetic properties of Er-doped GaN epilayer structures, grown by metal-organic chemical vapor deposition, with illumination from a light emitting diode. Single and multiple Er-doped epilayers were grown with Er concentrations up to $\sim 10^{21} \mathrm{~cm}^{-3}$. All samples exhibited hysteresis behavior at room temperature as measured by an alternating gradient magnetometer. When the samples were illuminated at a wavelength of $371 \mathrm{~nm}$, an increase in saturation magnetization was observed for each sample. The percentage increase for multiple layer samples ranged from 10\%-25\% indicating possible device applications. (C) 2009 American Institute of Physics. [DOI: 10.1063/1.3176972]
\end{abstract}

The optical properties of rare-earth (RE) doped semiconductors have been widely investigated for potential applications in telecommunication and full color display systems. ${ }^{1}$ When introduced into wide bandgap semiconductors, the excited states of trivalent RE ions (such as $\mathrm{Gd}, \mathrm{Tm}, \mathrm{Eu}$, and $\mathrm{Er}$ ) lead to sharp emission lines from the ultraviolet (UV) through the visible to the infrared (IR). ${ }^{2-4}$ Doping of AlGaN alloys with these same RE ions has also resulted in ferromagnetic behavior with Curie temperatures in excess of 300 $\mathrm{K}^{5-9}$ In addition, the concentrations of RE ions needed to induce ferromagnetism are significantly lower than those required for similar behavior with transition metals. In GaN films, concentrations of only $10^{16}-10^{18} \mathrm{~cm}^{-3}$ for $\mathrm{Gd}$, or $\sim 10^{19} \mathrm{~cm}^{-3}$ for Eu, are sufficient to produce ferromagnetic behavior. ${ }^{8,9}$ Furthermore, the electrical conductivity of the host nitride semiconductor can be controlled through codoping with conventional shallow level $n$-type dopants. ${ }^{10,11}$ Previously, we reported on the synthesis of Er-doped GaN (GaN:Er) films by metal-organic chemical vapor deposition (MOCVD) and the resulting optical and magnetic properties. ${ }^{12,13}$ In this paper we report on the change in the magnetic properties of Er-doped GaN epilayer structures, grown by MOCVD, with illumination at a wavelength of $371 \mathrm{~nm}$ from a light emitting diode.

The RE element Er possesses 11 electrons in the $4 f$ shell when it is in the trivalent charge state $\left(\mathrm{Er}^{3+}\right)$. The $4 f$ transitions in $\mathrm{Er}^{3+}$ between the first excited manifold $\left({ }^{4} I_{13 / 2}\right)$ and the ground state $\left({ }^{4} I_{15 / 2}\right)$ give rise to IR emissions near $1.54 \mu \mathrm{m}$. The importance of this wavelength region for optical communications has led to widespread research in Erdoped host materials such as silica fibers and various semiconductors including $\mathrm{Si}$. There are also $4 f$ transitions from the ${ }^{2} H_{11 / 2}$ and ${ }^{4} S_{3 / 2}$ levels to the ground state that lead to green emission for display applications. In addition, the three unpaired $4 f$ electrons in $\mathrm{Er}^{3+}$ can contribute to ferromagnetic

\footnotetext{
${ }^{a)}$ Electronic mail: nnepal@ncsu.edu.
}

ordering at room temperature (RT) as has been observed in GaN:Er films. ${ }^{13}$

The GaN:Er samples examined in this study were grown by MOCVD on (0001) $c$-plane sapphire substrates. The growth of these samples began with a thin GaN buffer layer, followed by a GaN epilayer template. ${ }^{12}$ Two different types of Er-doped GaN structures were realized: single epilayers of $\sim 0.5 \mu \mathrm{m}$ thickness, as shown in Fig. 1, and multilayer structures of the same thickness in which the Er growth was interrupted for time $t_{i}$. The Er concentration $\left(n_{\mathrm{Er}}\right)$ was in the range from $2-10 \times 10^{20} \mathrm{~cm}^{-3}$. Following MOCVD growth the samples were characterized by $\mathrm{x}$-ray diffraction (XRD) and photoluminescence (PL) measurements. The powder XRD spectra indicated high crystallinity and no second phase formation. Subsequently, small specimens $\left(2 \times 3 \mathrm{~mm}^{2}\right)$ were taken from each sample for magnetic characterization using an alternating gradient magnetometer (AGM).

The PL spectra measured at RT of a single epilayer and a multilayer with $t_{i}=194 \mathrm{~s}$ are displayed in Fig. 2. Both samples had $n_{\mathrm{Er}} \sim 2 \times 10^{20} \mathrm{~cm}^{-3}$. A laser diode at $370 \mathrm{~nm}$

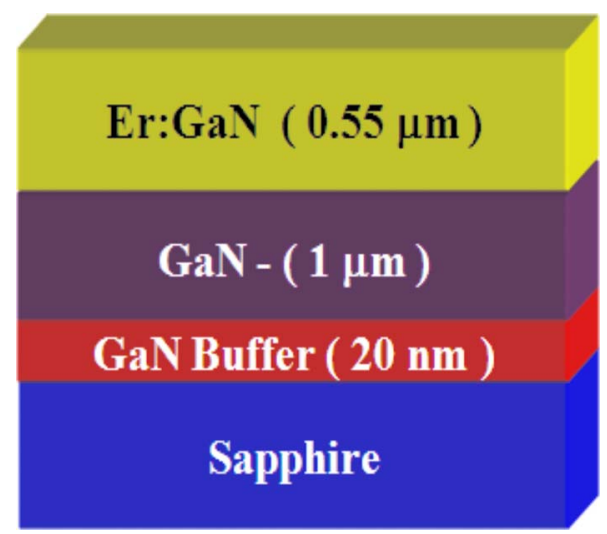

FIG. 1. (Color online) Schematic diagram of the typical GaN:Er structure examined in these experiments. 


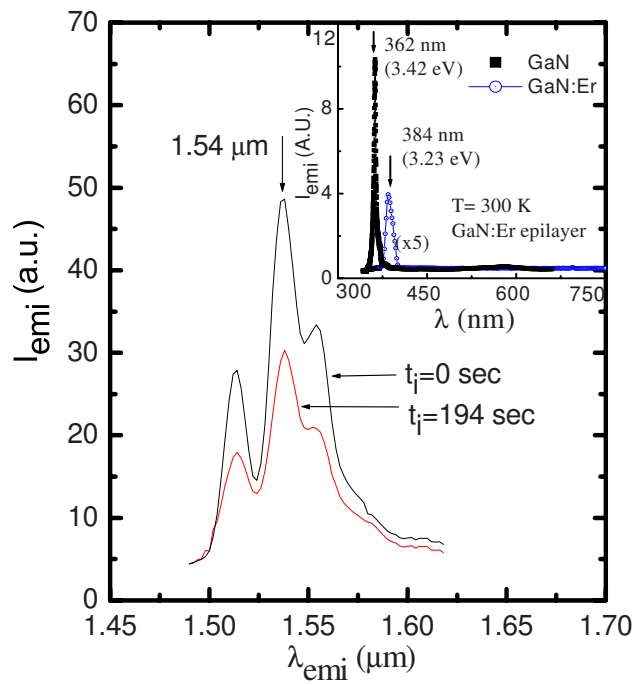

FIG. 2. (Color online) PL spectra taken at RT of the single GaN:Er epilayer and a multilayer sample with nearly the same Er concentration. A laser diode at $370 \mathrm{~nm}$ was the excitation source. The inset compares the band edge luminescence of a GaN:Er layer with that of an undoped epilayer.

was used for excitation and data were collected using a $0.33 \mathrm{~m}$ monochromator equipped with an InGaAs detector. Previously, it was found that a light emitting diode (LED) at a wavelength of $371 \mathrm{~nm}$ was very efficient for excitation of the $\mathrm{Er}^{3+}$ ions in GaN and InGaN epilayers. ${ }^{14}$ Each PL spectrum has the distinctive IR characteristics near $1.54 \mu \mathrm{m}$ of the $\mathrm{Er}^{3+}$ transitions between the ${ }^{4} I_{15 / 2}$ and ${ }^{4} I_{13 / 2}$ levels. The intensity of the PL spectrum from the single GaN:Er epilayer is $\sim 1.5 \times$ higher than that from the multilayer sample. This difference may be due to differences in material quality. With Er-doping of GaN epilayers there is a redshift of the band edge luminescence to $384 \mathrm{~nm}$ as shown in inset of Fig. 2 . Consequently, absorption of incident radiation at $370 \mathrm{~nm}$ in these samples should have been nearly equal.

Magnetic measurements were made at RT using an AGM with the magnetic field applied parallel to the sample surface. This is the easy axis of magnetization for the GaN:Er samples. A commercially available nitride LED (Nichia model NSHU550B) was used as the optical source during AGM measurements to determine the influence of illumination on the magnetic properties. The LED power output was $\sim 2 \mathrm{~mW}$ at $371 \mathrm{~nm}$ and the full width half maximum was $15-20 \mathrm{~nm}$. The GaN:Er samples were mounted at a distance of about $1 \mathrm{~mm}$ from LED's surface, as shown in the schematic diagram in Fig. 3. The diamagnetic properties of the substrate and holder were subtracted out and the data normalized to sample volume.

Without illumination (LED-off) all of the GaN:Er specimens displayed RT hysteretic behavior consistent with ferromagnetic ordering. However, the saturation magnetization $\left(M_{S}\right)$ of the different specimens varied considerably. In general, the observed $M_{S}$ of single GaN:Er epilayer samples was larger than that of multilayer samples. This result is consistent with the higher Er concentrations found in the single layer samples. With illumination (LED-on) there was an increase in $M_{S}$ for all samples.

In Fig. 4(a) hysteretic data taken at RT are presented for the single GaN:Er layer sample with $n_{\mathrm{Er}} \sim 2 \times 10^{20} \mathrm{~cm}^{-3}$ under LED-off and LED-on conditions. While the coercivity $\left(2 \mathrm{H}_{c}\right)$ remained constant, there was an increase in approxi-

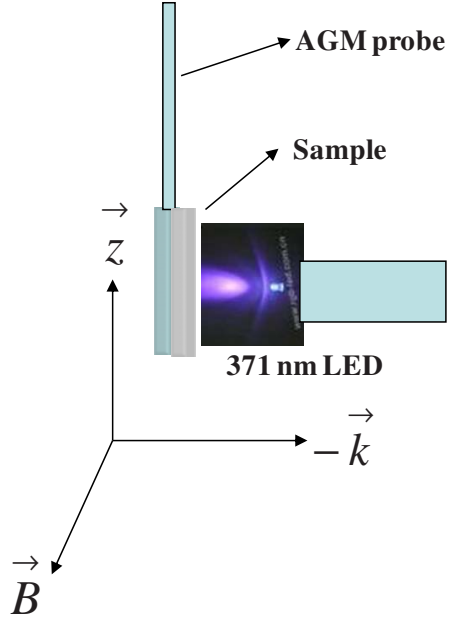

FIG. 3. (Color online) Illustration of the placement of the LED source and a GaN:Er sample for AGM measurements. The sample was at a distance of about $2 \mathrm{~mm}$ from LED's surface.

mately $0.8 \mu \mathrm{emu}$ in $M_{S}$ when the sample was illuminated. The measured increase, $\Delta M_{S}=M_{S}$ (on)- $M_{S}$ (off), for each of the single layer samples is plotted in Fig. 4(b) as a function of Er concentration. Also plotted are the $M_{S}$ (off) values. The largest increase was observed for the sample with the lowest Er concentration. The other samples had a higher initial $M_{S}$ value but a slightly lower $\Delta M_{S}$ of $\sim 0.7 \mu \mathrm{emu}$. The GaN:Er multilayer sample had a similar response to LED illumina-
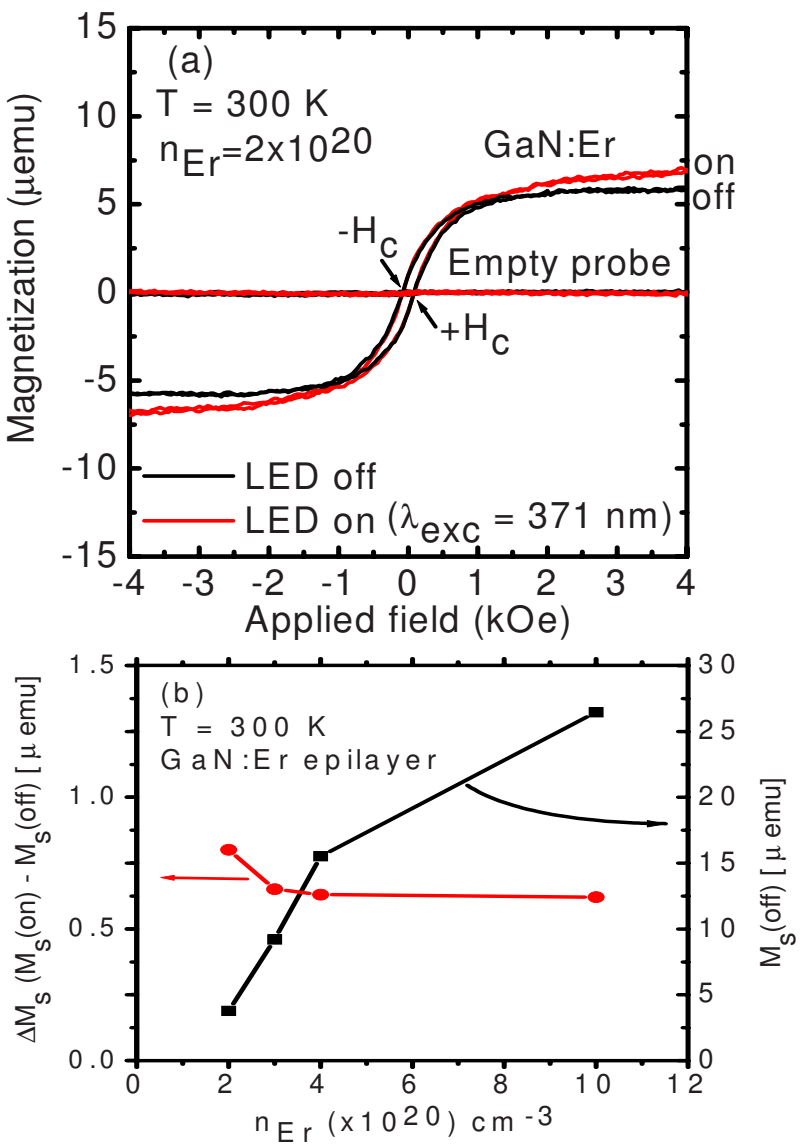

FIG. 4. (Color online) (a) Hysteretic data taken at RT for a GaN:Er single layer sample with $n_{\mathrm{Er}} \sim 2 \times 10^{20} \mathrm{~cm}^{-3}$ under LED-off and LED-on conditions. (b) The measured $\Delta M_{S}$ and $M_{S}$ (off) values for the single layer samples plotted as a function of Er concentration. 


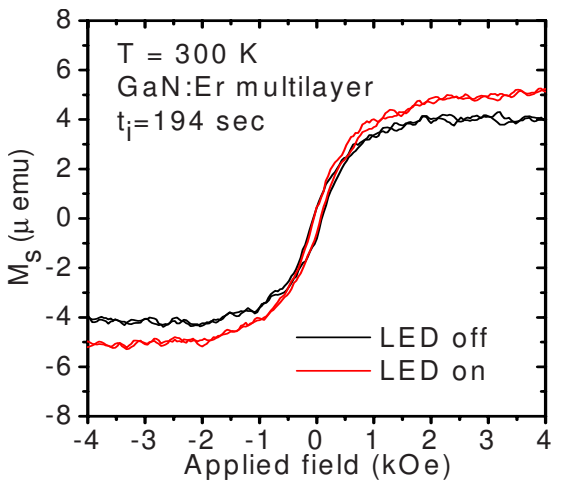

FIG. 5. (Color online) (a) Hysteretic data taken at RT for a GaN:Er multilayer sample with $n_{\mathrm{Er}} \sim 2 \times 10^{20} \mathrm{~cm}^{-3}$ and time interruption of $194 \mathrm{~s}$.

tion. As shown in Fig. 5, the sample with $t_{i}=194 \mathrm{~s}$ yielded good hysteretic behavior under LED-off conditions. With illumination, the measured $\Delta M_{S}$ was $\sim 1.0 \mu \mathrm{emu}$, which is $\sim 25 \%$ increase. The measured coercivity was constant under LED off/on conditions. After illumination, with LED-off, the saturation magnetization returned immediately to original levels.

The results of these experiments provide a greater understanding of the mechanism leading to RT ferromagnetic ordering in RE-doped III-N semiconductor films. In all GaN:Er samples, illumination by a LED produced an increase in the measured $M_{S}$. As shown in Fig. 4(b) this increase was dependent upon the Er concentration. Samples with a lower level of Er-doping, e.g., $n_{\mathrm{Er}} \sim 2 \times 10^{20} \mathrm{~cm}^{-3}$, had a lower measured $M_{S}$ but a larger $\Delta M_{S}$. With samples having higher $n_{\mathrm{Er}}$ levels, the measured $M_{S}$ (off) was greater but the $\Delta M_{S}$ remained nearly constant. The data indicate that electron hole pairs created by incident photons from the LED source facilitate ferromagnetic ordering in GaN:Er layers. This suggests a carrier-mediated mechanism ${ }^{15}$ for the RT ferromagnetism observed in these Er-doped films. From the present experiments it is unclear whether the magnetic ordering is due to positive or negative carriers. However, since $\Delta M_{S}$ was constant for the higher $n_{\mathrm{Er}}$ levels, the photon flux of the LED may have been the limiting factor in these experiments. Use of a laser source may have produced a larger increase in $M_{S}$.

The magnetic data shown in Fig. 5 for the multilayer structure had a similar behavior. Since the Er level was $\sim 2$ $\times 10^{20} \mathrm{~cm}^{-3}$, the measured $M_{S}$ (off) value was nearly that of the comparably doped single epilayer. With illumination the observed $\Delta M_{S}$ was slightly higher but equal within experimental accuracy. These results indicate that GaN:Er multilayer structures may be fabricated with nearly the same magnetic properties.
In summary, we have shown that illumination of Erdoped GaN epilayer structures at a wavelength of $371 \mathrm{~nm}$ from a LED leads to an enhancement of magnetic properties. Electron hole pairs created by incident photons from the LED facilitate RT ferromagnetic ordering in the GaN:Er layers, suggesting a carrier-mediated mechanism. Similar results may occur for other RE-doped III-N semiconductor films. Furthermore, the magnetic response of single and multiple Er-doped GaN epilayers yielded similar optical and magnetic properties results when Er concentrations were the same.

The authors thank Dr. N. El-Masry for her helpful comments concerning this work, which was performed under grants from the U.S. Army Research Office (Grant No. W911NF-06-1-0134) and the National Science Foundation (Grant No. EECS-0823894). J.Y.L. and H.X.J. gratefully acknowledge the support of the Linda Whitacre and Edward Whitacre endowed chair positions through the AT\&T Foundation.

N. Nepal is a fellow of NRC-ARO Postdoctoral Associateship Program.

${ }^{1}$ See for example, H. Ennen, J. Schneider, G. Pomrenke, and A. Axmann, Appl. Phys. Lett. 43, 943 (1983); A. J. Steckl and J. M. Zavada, MRS Bull. 24, 33 (1999); A. J. Steckl, J. H. Park, and J. M. Zavada, Mater. Today 10, 20 (2007).

${ }^{2}$ S. Hufner, Optical Spectra of Transparent Rare Earth Compounds (Academic, New York, 1978).

${ }^{3}$ A. J. Steckl, J. Heikenfeld, M. Garter, R. Birkhahn, and D. S. Lee, Compound Semicond. 6, 48 (2000).

${ }^{4}$ J. M. Zavada, N. Nepal, J. Y. Lin, H. X. Jiang, E. Brown, U. Hömmerich, J. Hite, G. T. Thaler, C. R. Abernathy, S. J. Pearton, and R. Gwilliam, Appl. Phys. Lett. 89, 152107 (2006).

${ }^{5}$ N. Teraguchi, A. Suzuki, Y. Nanishi, Y.-K. Zhou, M. Hashimoto, and H. Asahi, Solid State Commun. 122, 651 (2002).

${ }^{6}$ H. Bang, J. Sawahata, G. Piao, M. Tsunemi, H. Yanagihara, E. Kita, and K. Akimoto, Phys. Status Solidi C 0, 2874 (2003).

${ }^{7}$ S. Dhar, O. Brandt, M. Ramsteiner, V. F. Sapega, and K. H. Ploog, Phys. Rev. Lett. 94, 037205 (2005).

${ }^{8}$ S. Dhar, T. Kammermeier, A. Ney, L. Perez, K. H. Ploog, A. Melnikov, and A. D. Wieck, Appl. Phys. Lett. 89, 062503 (2006).

${ }^{9}$ J. Hite, G. T. Thaler, R. Khanna, C. R. Abernathy, S. J. Pearton, J. H. Park, A. J. Steckl, and J. M. Zavada, Appl. Phys. Lett. 89, 132119 (2006).

${ }^{10}$ J. K. Hite, R. M. Frazier, R. P. Davies, G. T. Thaler, C. R. Abernathy, S. J. Pearton, J. M. Zavada, E. Brown, and U. Hömmerich, J. Electron. Mater. 36, 391 (2007).

${ }^{11}$ R. Wang, A. J. Steckl, E. E. Brown, U. Hommerich, and J. M. Zavada, J. Appl. Phys. 105, 043107 (2009).

${ }^{12}$ C. Ugolini, N. Nepal, J. Y. Lin, H. X. Jiang, and J. M. Zavada, Appl. Phys. Lett. 89, 151903 (2006); 90, 051110 (2007).

${ }^{13}$ J. M. Zavada, N. Nepal, C. Ugolini, J. Y. Lin, H. X. Jiang, R. Davies, J. Hite, C. R. Abernathy, and S. J. Pearton, Appl. Phys. Lett. 91, 054106 (2007)

${ }^{14}$ R. Dahal, C. Ugolini, J. Y. Lin, H. X. Jiang, and J. M. Zavada, Appl. Phys. Lett. 93, 033502 (2008).

${ }^{15}$ C. Zener, Phys. Rev. 81, 440 (1951); 83, 299 (1951). 